\title{
Adaptive Precision Solvers for Sparse Linear Systems
}

\author{
Hartwig Anzt \\ Innovative Computing Lab \\ University of Tennessee \\ Knoxville, Tennessee, USA \\ hanzt@icl.utk.edu
}

\author{
Jack Dongarra \\ Innovative Computing Lab \\ University of Tennessee \\ Knoxville, Tennessee, USA \\ dongarra@icl.utk.edu
}

\author{
Enrique S. Quintana-Ortí \\ Depto. Ingeniería y Ciencia de \\ Computadores \\ Universidad Jaume I \\ Castellón, Spain \\ quintana@icc.uji.es
}

\begin{abstract}
We formulate an implementation of a Jacobi iterative solver for sparse linear systems that iterates the distinct components of the solution with different precision in terms of mantissa length. Starting with very low accuracy, and using an inexpensive test, our technique extends the mantissa length for those component updates when and where this is required. Numerical experiments reveal that, for a solver that pursues IEEE double precision accuracy in the solution (i.e., mantissa of 52 binary digits), the precision required to reach convergence for the distinct components can differ significantly during the iteration so that, during most of this process, only a few components may require operating with the full length of the mantissa. Thus, with operations involving a longer mantissa yielding a higher power usage, energy savings can potentially be obtained by using a truncated format. Finally, we introduce a novel metric which quantifies the average mantissa length during the iteration, and exposes the resource savings of the Jacobi solver with adaptive mantissa.
\end{abstract}

\section{CCS Concepts}

-Mathematics of computing $\rightarrow$ Solvers; -Theory of computation $\rightarrow$ Massively parallel algorithms; • Hardware $\rightarrow$ Hardware accelerators;

\section{Keywords}

Sparse linear systems, iterative solvers, Jacobi method, variable precision, computer arithmetic.

\section{INTRODUCTION}

Power and energy presently pose two major obstacles to build faster computer architectures. Concretely, the end of Dennard's scaling [12], combined with fixed power budgets, have resulted in the power dissipation capacity of CMOS technology severely constraining the number of transistors that can be active in current chips [14, 16, 29]. In addition, energy consumption exerts pressure on large supercomputing facilities, in terms of economic costs [20,

Permission to make digital or hard copies of all or part of this work for personal or classroom use is granted without fee provided that copies are not made or distributed for profit or commercial advantage and that copies bear this notice and the full citation on the first page. Copyrights for components of this work owned by others than ACM must be honored. Abstracting with credit is permitted. To copy otherwise, or republish, to post on servers or to redistribute to lists, requires prior specific permission and/or a fee. Request permissions from Permissions@ acm.org.

E2SC15 November 15-20, 2015, Austin, TX, USA

(C) 2015 ACM. ISBN 978-1-4503-3994-0.. \$15.00

DOI: http://dx.doi.org/10.1145/2834800.2834802
21], but also at a much smaller scale, for example by reducing the battery lifetime of hand-held (mobile) devices.

Dark silicon (i.e., keeping only the necessary parts of the chip active) is currently fueling the development and adoption of heterogeneous architectures equipped with hardware accelerators (coprocessors), such as AMD and NVIDIA's graphics processing units (GPUs) or the Intel Xeon Phi [1]. Following this trend, applicationspecific coprocessors will eventually become the only path to keep up with the advances in computing performance predicted by Moore's Law [23]. However, for specialization to be a viable path to leverage dark silicon, it must provide reasonable acceleration and energy savings over a wide range of workloads, thus favoring the adoption of reconfigurable accelerators based on FPGAs (field programmable gate arrays) [26].

Approximate computing (AC) has been proposed as a means to tackle the "utilization wall," by focusing only on those parts of the computation that have a visible effect on the quality of the result, thus trading off accuracy (and/or reliability) for energy consumption [22, 24, 27]. However, AC is, in general, inappropriate for numerical computations, where high accuracy is usually a must, and tiny errors can easily propagate to produce a useless solution.

In this paper, we consider the numerical solution of sparse linear systems via iterative algorithms. Concretely, we focus on the Jacobi method [25], a component-wise relaxation (i.e., stationary) solver of appealing simplicity and parallelism. This type of iterative method naturally embeds a certain degree of error resilience in case of hardware outage or a low error rate [5, 3]. In our work, we exploit this fault tolerance differently, in order to formulate a specialized solver that can operate with component-wise variable precision. Specifically, our solver initially iterates with a very short mantissa, but relies on a light-weight test to adaptively extend its length component-wise, as it becomes necessary. By operating with reduced (adaptive) precision, we can expect a potential delay in the iteration convergence. However, the hope in this AC adaptive precision approach is that, with the utilization of longer mantissas drawing higher power consumption, energy savings can still be obtained by eventually implementing this technique in an energy-efficient FPGA circuit with multiple precision formats.

The rest of the paper is structured as follows. In Section 2 we describe related work. In Section 3 we briefly review the stationary solvers, focusing on the Jacobi method. This is followed by the major contributions of our paper: In Section 4 we derive an adaptivemantissa scheme for the Jacobi method; and in Sections 5-6 we experimentally evaluatate the convergence and the quantification of energy savings via a novel metric that captures the aggregated number of mantissa binary digits (bits) that can be saved with our technique. Finally, we close the paper in Section 7 with a number of concluding remarks and a discussion of future work. 


\section{RELATED WORK}

AC has recently been proposed as a means to trade off accuracy for energy in general-purpose applications, thus helping to address utilization and energy challenges. The key is that many applications naturally embed a certain degree of tolerance to "errors," which can be leveraged to reduce the energy that is usually spent to guarantee correctness. For example, in image rendering, it may be reasonable to accept a small number of erroneous pixels from a video decoder in return for extended battery life. Other examples include probabilistic inference applications, audio signal processing, service profiling, Monte Carlo simulations, and machine learning algorithms; see $[22,27]$ and the references therein.

The effect of rounding errors and finite precision arithmetic on LA methods has been studied extensively in the recent decades. The general conclusion is that, during the operation of a numerically stable algorithm, tiny rounding errors in the floating-point arithmetic can accumulate to produce a solution with a quality that is bounded by the precision arithmetic and the conditioning of the problem [17]. For dense and sparse LA computations, the current practice is to perform all computations in double precision. The only significant exception is the use of mixed single-double precision arithmetic, in combination with iterative refinement, which can be, for example, applied in direct and iterative solvers for dense and sparse linear systems to produce a solution with double precision accuracy, at roughly the cost of operating with single precision arithmetic [4, 6, 7].

Some recent work [15] proposes a significance-driven execution of the Jacobi method that reduces energy consumption via near threshold voltage computing (NTVC). This approach integrates an execution scheme that switches between highly concurrent nearthreshold (error-prone) execution and sequential above-threshold (error-free) execution, as the iteration converges. This technique thus points in the direction of a connection between $\mathrm{AC}$ and fault tolerance for soft (transient) errors, but operates at the level of the complete solver, applying a criterion that is too coarse to yield a practical switching point for the Jacobi method.

For the iterative solution of sparse linear systems, our work explicitly exposes the link between AC and fault tolerance. For example, in the event of bit-flips that corrupt the result from one or more arithmetic operations, fault tolerance aims to deliver a "correct" solution (as exact as possible with respect to the conditioning and the precision arithmetic). Compared with this, our proposal for $\mathrm{AC}$ also pursues convergence to the correct solution, but employs different precision levels during the iteration, which can be roughly viewed as errors introduced in the digits that are "chopped" during the computations. This insight paves the road to exploit AC in combination with recent advances in the development of fault-tolerant iterative solvers for sparse linear systems [8, 18, 13, 28].

Following this idea, in this paper we revisit our previous work on fault tolerance for the Jacobi method [3] to elaborate on a version of this solver that adapts the precision of the computations where and when it is necessary. Our work thus provides a solution that is both more flexible and more powerful than a simple mixed precision approach, providing a common framework to tackle AC and fault tolerance in the iterative solution of sparse linear systems via the Jacobi method.

\section{STATIONARY METHODS}

Let us consider the linear system $A x=b$, where $A \in \mathbb{R}^{n \times n}$ is a sparse matrix, $b \in \mathbb{R}^{n}$ denotes the right-hand side vector, and $x \in \mathbb{R}^{n}$ contains the solution. Stationary solvers, such as the Jacobi and Gauss-Seidel iterations [25], apply component-wise relaxation to update, at each iteration $k$, each individual component of an approximate solution $x^{\{k\}}$, with respect to $A, b$, and $x^{\{k-1\}}$. In the particular case of the Jacobi method, this update can be explicitly formulated as:

$$
\begin{aligned}
x^{\{k\}} & :=D^{-1}\left(b-(A-D) x^{\{k-1\}}\right) \\
& =D^{-1} b+M x^{\{k-1\}}, \quad k=1,2, \ldots,
\end{aligned}
$$

where $D \in \mathbb{R}^{n \times n}$ is a diagonal matrix that only contains the diagonal entries of $A$, and $x^{\{0\}}$ corresponds to a starting solution guess [25].

An appealing property of the Jacobi method is that all components of $x^{\{k\}}$ can be obtained in parallel, as the update on any component $x_{i}^{\{k\}}$ involves values from $x^{\{k-1\}}$ only. In contrast, the Gauss-Seidel method requires values from both the last and the current iterate, imposing a specific updating order that strongly constrains its concurrency.

From the numerical perspective, the Jacobi iteration converges if the spectral radius of the iteration matrix $M$ is smaller than one [25]. This turns a Jacobi-based solver into an appealing approach for a variety of problems, including the approximate solution of sparse triangular solves in the context of iterative ILU preconditioning [10, 2].

To close this short review, we note that the adaptive-mantissa version of the Jacobi method that we propose in this work preserves the convergence property of the original method, with the only limitation being a potential decrease in the convergence rate. In particular, the Jacobi iteration itself remains untouched, and the implementation is only extended with an additional test for the contraction property at the component level. This is used to identify those components of $x^{\{k\}}$ for which the following iterations have to be handled with a higher precision format.

\section{MANTISSA-ADAPTIVE FORMULATION OF THE JACOBI METHOD}

\subsection{Component-wise mantissa extension}

We open this section by noting that, in the IEEE 754 doubleprecision binary floating-point format, the sign takes $1 \mathrm{bit}$; the exponent occupies 11 bits; and the "precision" of the significand (or mantissa) is 53 bits, with only 52 of these bits explicitly being stored. Overall, this offers a precision of 15-17 significant decimal digits [19].

The concept we use to dynamically adapt the mantissa length is similar to the fault detection strategy proposed in [3]. Starting with the solver operating with a very short mantissa for all components, our approach checks whether the mantissa should be extended for a certain component by exploiting the property that, in a synchronous relaxation method such as Jacobi, the contraction property is fulfilled component-wise. Concretely, this property establishes that, for any component of the approximate solution vectors at iterations $k$ and $k-1$,

$$
\begin{aligned}
& \exists 0<\theta_{i}<1: \\
& \left|x_{i}^{\{k\}}-x_{i}^{\{k-1\}}\right| \leq \theta_{i}\left|x_{i}^{\{k-1\}}-x_{i}^{\{k-2\}}\right| \leq \theta_{i}^{2}\left|x_{i}^{\{k-2\}}-x_{i}^{\{k-3\}}\right| \ldots
\end{aligned}
$$

Furthermore, due to the linear convergence rate of the Jacobi iteration, the ratios

$$
c_{i}^{\{k\}}:=\frac{z_{i}^{\{k-1\}}}{z_{i}^{\{k\}}}=\frac{\left|x_{i}^{\{k-1\}}-x_{i}^{\{k-2\}}\right|}{\left|x_{i}^{\{k\}}-x_{i}^{\{k-1\}}\right|}, \quad k \geq 2,
$$


are, in general, different for the distinct components, but they remain constant up to convergence; i.e., $c_{i}^{\{2\}}=c_{i}^{\{3\}}=c_{4}^{\{i\}}=\ldots=c_{i}$, where we note that $c_{i}>1$ is necessary for convergence.

The key to our approach is that in an execution with a certain precision arithmetic, deviations from this contraction constant $c_{i}$ indicate that this component has converged in the "current" format. More precisely, an exploding ratio $z_{i}^{\{k-1\}} / z_{i}^{\{i\}}$ due to a very small $z_{i}^{\{k\}}$ serves as an indication that the approximation for this component has converged for the system considered in the current precision. If that is the case, in the adaptive-mantissa version of the Jacobi method, the mantissa length is extended for this component. In a strongly coupled system, stagnation of a component might require higher precision for another, adjacent component. However, in a case where extending the component's precision does not resolve the problem, stagnation will propagate through the system, and eventually the adjacent component will also be extended.

To reduce the cost of the test, one can search for deviations from the contraction constant, not after every iteration, but at a lower pace. This is equivalent to considering a test based on the deviations between the ratio $z_{i}^{\{k-\phi\}} / z_{i}^{\{k\}}$ and $c_{i}^{\phi}$, where the integer $\phi \geq 1$ determines the periodicity of the test. Now, the use of limited precision can be expected to introduce rounding effects that result in variations from the expected linear convergence rate. To account for these, a test which determines whether a component needs to iterate with a higher precision (i.e., longer mantissa length) could rely on a tolerance-based estimation of the difference. Concretely, for some threshold $\tilde{\delta}$, the extension condition

$$
\left|\frac{z_{i}^{\{k-\phi\}}}{z_{i}^{\{k\}}}-c_{i}^{\phi}\right|>\tilde{\delta}
$$

can be used to determine that a mantissa extension is necessary.

In practice, we bound this threshold by setting

$$
\tilde{\delta}:=\delta \cdot\left(c_{i}^{\phi}-1\right)
$$

for some user-defined $0<\delta<1$. This ensures that stagnation, as indicated by $z_{i}^{\{k-\phi\}} / z_{i}^{\{k\}} \approx 1$, is never accepted, especially not when the sequence $z_{i}^{\{0\}}, z_{i}^{\{1\}}, z_{i}^{\{2\}}, \ldots$ smoothly approaches zero without violating the threshold condition.

In the application of this convergence check, the threshold controls how quickly the mantissa is extended: For small values of $\delta$, minor deviations from the expected convergence rapidly trigger a mantissa extension; conversely, for large values of this parameter, the mantissa is extended only very gradually as the deviations need to be quite significant to induce an extension. Consequently, small thresholds can be expected to result in a convergence rate that is only slightly different from that of the Jacobi method operating with full precision, while large thresholds may typically result in a larger convergence delay. In case stagnation is detected, and the mantissa was extended for at least one of the components, the next iteration has to propagate the information made available via the higher accuracy, and should not trigger additional mantissa extension. Also, the subsequent iteration should be non-relevant for a mantissa extension, as the ratios $z_{i}^{\{k-\phi\}} / z_{i}^{\{k\}}$ need to realign. In summary, every mantissa extension is followed by two iterations where the components are updated without checking for stagnation. This can become a bottleneck for problems with fast convergence.

From equation (5) it is obvious that larger precision-related deviations from the contraction constant $c_{i}$ can be tolerated for larger differences from $c_{i}^{\phi}-1$. Hence, for an efficient realization, the periodicity of the test (i.e. the constant $\phi$ ) should be reasonably large. This also helps to reduce the overhead of the test. The disadvantage is that a low frequency can "waste" a high number of iterations. Alternatively, arbitrary thresholds can be chosen in the extension condition (4) by explicitly excluding the value 1 . However, this results in a more complex extension test, and increased computational cost.

\subsection{Implementation details}

A practical detail that is important to note is that the first iteration cannot be used to derive the contraction constant rates $c_{i}$ in (5). For example, for a component where the matrix row contains a non-zero only on the diagonal, the exact solution is generated by the first update, and this component remains unmodified during the iteration. Typically, obtaining the contraction constants from the third or fourth iteration provides appropriate values. In practice this means that the initial 1-3 iterations are performed in full precision (i.e., using a mantissa of full length) to obtain exact values for $c_{i}$.

The application of the threshold test every $\phi>1$ iterations tolerates larger rounding effects by choosing a larger threshold, as explained in Section 4.1. Also, when this condition is violated, we extend the mantissa length for the corresponding component by $\gamma$ binary digits. The parameters $\gamma$ and $\phi$ should be adapted to the target system: If the first iterations in full precision expose a fast convergence rate, the frequency should be increased (smaller $\phi$ ), and/or the extensions should be substantial (larger $\gamma$ ). The implementation of this strategy for a user-defined threshold $\delta$ is outlined in Figure 1 using MATLAB $\AA$ code. There, the variable $f l$ ag is used to ignore convergence perturbations in the two iterations following a mantissa extension.

\section{EXPERIMENTAL EVALUATION}

\subsection{Setup and solvers}

All the experiments in this section were performed using MATLAB (release R2014a) and IEEE 754 double-precision (64-bit) arithmetic, on a server equipped with two Intel Xeon E5-2670 sockets $(2 \times 16$ cores, operating at $2.6 \mathrm{GHz})$ and $64 \mathrm{~GB}$ of RAM.

For reference, in the evaluation we include two standard implementations of the Jacobi method, operating with full precision (mantissa of 52 bits) and a cropped format where the mantissa is truncated to 8 bits respectively. Hereafter, we will refer to these two respective versions as the full-mantissa Jacobi (or simply, Jacobi) and the 8bit-mantissa Jacobi. Obviously, the latter will typically not converge to a solution with high accuracy, but rather stagnate at a certain level. (In general, the 8bit-mantissa Jacobi could also diverge in case the perturbed system does not fulfill the contraction property.) Our adaptive-mantissa version of Jacobi is also started with a mantissa length of 8 binary digits, but this value is extended as dictated by the test (4)-(5) for the distinct components. This accuracy is sufficient for the iteration matrix to fulfill the contraction property. The default gradient used by the adaptive-mantissa Jacobi to extend the mantissa is $\gamma=8$, but similar results were observed for $\gamma=4$. We note that there exits an interaction among $\delta$, $\phi$, and $\gamma$, as they modulate the "speed" with which the precision is extended.

\subsection{Benchmark problem and convergence cri- terion}

The test problem is a 27-pt stencil discretization of the 3D Laplace problem using a uniform $16 \times 16 \times 16$ mesh. This results in a system matrix of size 4,096 with 97,336 nonzeros and a condition number of 93.6. 


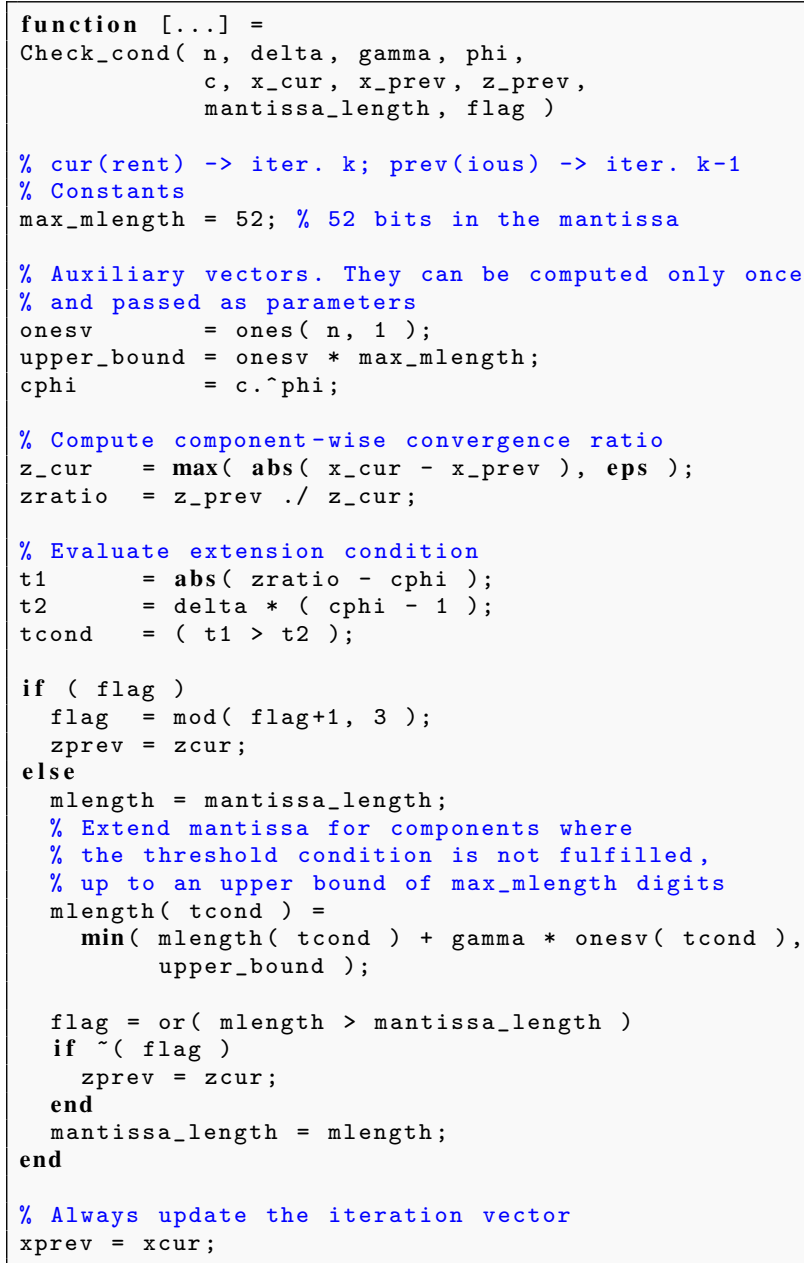

Figure 1: Mantissa-adaptive strategy in MATLAB, applied every phi $=\phi$ iterations.

The solvers use a right-hand side vector $b$ with all its components set to 1 , and commence with the initial starting guess $x^{\{k\}}=0$. The convergence and stopping criterion are based on the relative residual norm,

$$
\mathscr{R}\left(x^{\{k\}}\right)=\frac{\left\|b-A x^{\{k\}}\right\|_{2}}{\left\|b-A x^{\{0\}}\right\|_{2}}=\frac{\left\|b-A x^{\{k\}}\right\|_{2}}{\|b\|_{2}}
$$

being smaller than a certain convergence threshold $\tau$.

\subsection{Simulation of adaptive precision hardware}

For our experimental analysis of the adaptive-mantissa Jacobi, the hardware that handles the distinct components with different accuracy (adaptive mantissa length) is emulated as follows. At iteration $k$, for a component $i$ that is to be computed with a mantissa of length $\mathscr{L}_{i}^{\{k\}}$ :

1. All the (nonzero) values in the $i$-th row of the coefficient matrix $A$, the approximation $x^{\{k-1\}}$, and the corresponding component of the right-hand side $b_{i}$, are copied into auxiliary variables, $\tilde{a}^{T}, \tilde{x}^{\{k-1\}}$ and $\tilde{b}_{i}$ respectively, where their $52-\mathscr{L}_{i}^{\{k\}}$ less significant bits are set to zero.

2. The dot product $y_{i}:=\tilde{a}^{T} \cdot \tilde{x}^{\{k-1\}}$ is computed using the truncated vectors and 64-bit arithmetic. The scalar result $y_{i}$ from this operation is then truncated to $\tilde{y}_{i}$ by setting its $52-\mathscr{L}_{i}^{\{k\}}$ less significant bits to zero.

3. Next, $p_{i}:=\left(\tilde{b}_{i}-\tilde{y}_{i}\right) / \tilde{a}_{i}^{T}$ is computed in 64-bit arithmetic and truncated to $\tilde{p}_{i}$.

4. To complete the process, the next iterate $x_{i}^{\{k\}}:=x_{i}^{\{k-1\}}+\tilde{p}_{i}$ is computed using 64-bit arithmetic and the result is finally truncated to the desired precision.

For the 8bit-mantissa Jacobi, $\mathscr{L}_{i}^{\{k\}}=8$ during the complete iteration.

The reason to enforce this emulated mode is that our hardware does not support the necessary range of mantissa formats, and truncating the output of all floating-point operations (flops), via software, during the simulation is too expensive. We recognize that, mathematically, our "dot-based" emulated truncation, which operates at the level of the dot product that updates a given component $x_{i}^{\{k\}}$, is not identical to using an actual truncated format for all the computations (i.e., truncating per flop). However, we expect that the differences are similar to those resulting from the application of different reduction schemes in the accumulation for the dot products.

Figure 2 compares, for a smaller variant of the Laplace benchmark ( $4 \times 4 \times 4$ grid with $n=64)$, the behaviour of the dot-based emulated truncation strategy and an actual execution where all flops are truncated to the desired precision ("flop-based" truncated precision processor). The left-hand side plots report the relative residual $\mathscr{R}\left(x^{\{k\}}\right)$ as the iteration progresses, showing close convergence rates for both variants, independently of the value $\delta$. The right-hand side plot summarizes the number of bits employed by the adaptive-mantissa Jacobi with dot-based and flop-based truncations, at each iteration, into three values: minimum, maximum, and average. Let us assume that, at iteration $k$, these values are respectively $\min ^{\{k\}}, \max ^{\{k\}}$ and $\operatorname{avg}^{\{k\}}$. This means that the updates of all components in $x^{\{k\}}$ employ between $\min ^{\{k\}}$ and $\max \{k\}$ mantissa bits, and on average they utilize $\operatorname{avg}^{\{k\}}$ bits. Note that these plots reflect a scenario where the initial 3 iterations are performed in full precision to obtain $c_{i}$. In addition, we can observe a small gap in favor of the dot-based emulated truncation strategy from the point of views of all three bit quantification metrics, larger for $\delta=0.9$, which can be judged to be around 5 bits for the average number of bits.

\subsection{Selecting the tolerance threshold $\delta$}

The plots in the left-hand side of Figure 3 compare the convergence rates of the two reference versions (full-mantissa Jacobi and 8bit-mantissa Jacobi) with that of the adaptive-mantissa Jacobi, for different values of the threshold $\delta$, and the $(16 \times 16 \times 16) 3 \mathrm{D}$ Laplace problem. The right-hand side plots in the figure visualize the variation of the minimum, maximum and average mantissa lengths for the adaptive-mantissa Jacobi across the iteration process. The plots on both sides also indicate the iterations where, for the adaptive variant, the mantissa of at least one component is extended by $\gamma=8$ additional digits.

The plots on the left-hand side of the figure show that the 8bitmantissa Jacobi does not converge, but stagnates at a certain residual level which is conformal with the constrained precision employed in this version. Conversely, the adaptive version presents minor convergence deviations from the Jacobi implementation using full precision for all three values of the extension threshold $\delta$, with a gap that grows slightly with this parameter. This appealing behavior of the adaptive-mantissa Jacobi comes from a steady extension of the mantissa during the iteration, very rapid for $\delta=0.1$, 


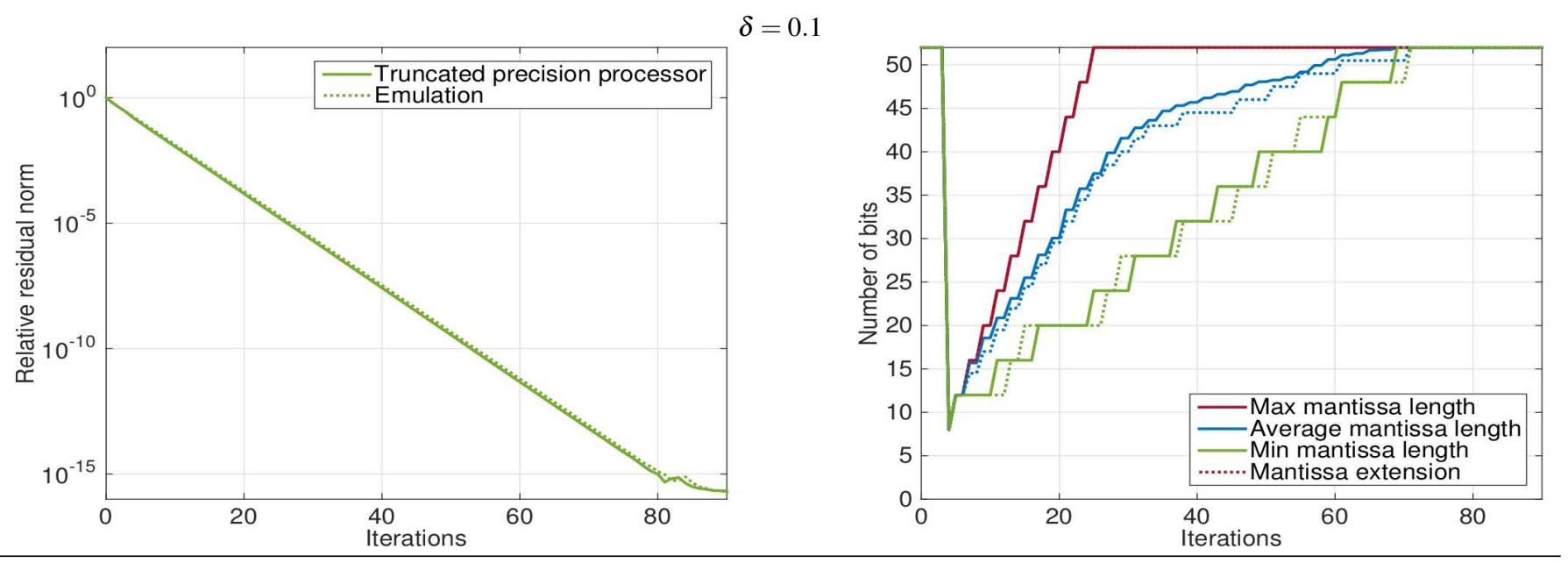

$\delta=0.9$
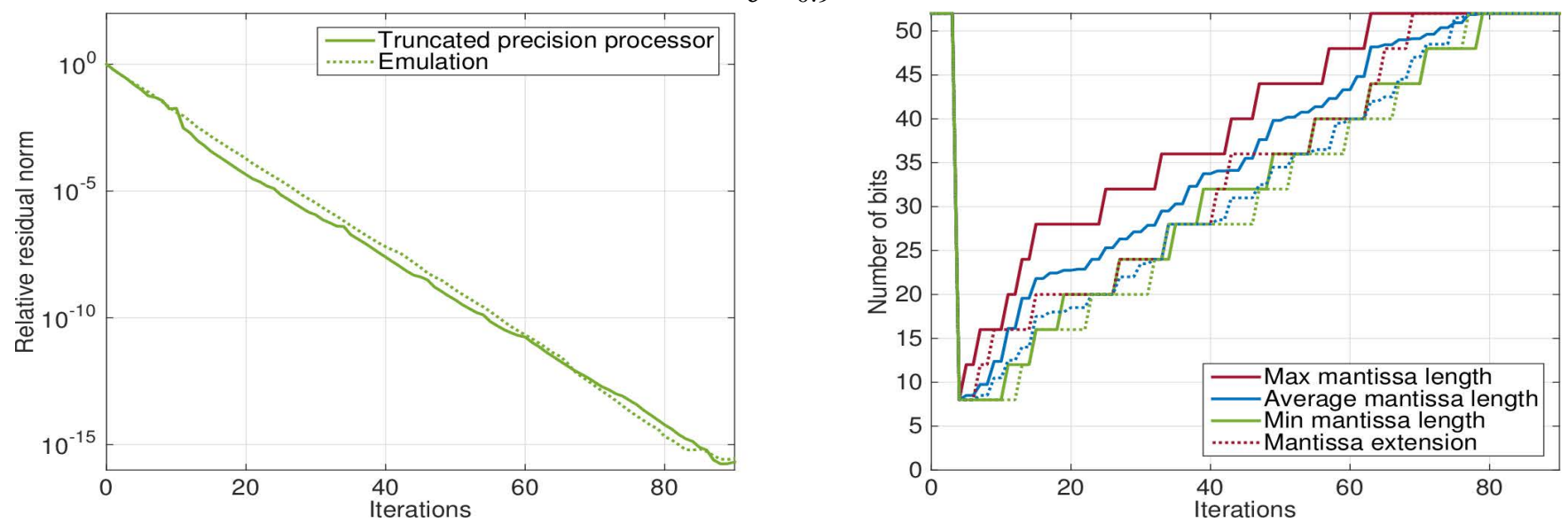

Figure 2: Convergence (left) and behavior of the mantissa length (right) for the adaptive-mantissa Jacobi using (the appropriate) truncated precision for all flops (solid lines), and the emulated truncation strategy that operates at the level of the dot products only (dotted lines). The test case is a 27-pt stencil discretization of the 3D Laplace problem using a $4 \times 4 \times 4$ grid. For this experiment, $\phi=1$ and the mantissa was extended by $\gamma=4$ bits when required by the threshold test using $\delta=0.1$ (top) and $\delta=0.9$ (bottom).

and slower for the remaining two threshold values. For the smallest value of $\delta$, the average mantissa length in the adaptive-mantissa iteration is extended close to full precision at about iteration 200. Compared to this, the results obtained with $\delta=0.5$ and 0.9 demonstrate that it is possible to operate most of the time with a much shorter average mantissa, and still attain (almost) the same level of accuracy in the intermediate and final residuals in about the same number of iterations.

In order to select the best value for $\delta$ as well as assess the actual savings that the adaptive-mantissa Jacobi renders, up to a certain iteration/convergence residual, we propose to quantify the computational cost, and therefore the potential savings, in terms of the number of mantissa bits (resources) that are "consumed" up to that point. For iteration $k$, we define the computational cost from the combination of nonzeros in the distinct rows of $A$, say $n n z_{i}(A)$, with the mantissa formats used in this iteration for each component:

$$
\mathscr{C}^{\{k\}}:=\sum_{i=1}^{n} n n z_{i}(A) \cdot \mathscr{L}_{i}^{\{k\}} .
$$

Thus, assuming the desired relative residual stopping criterion has been reached at iteration $\tilde{k}$, we can define the total computational cost as:

$$
\mathscr{C}^{[1, \tilde{k}]}:=\sum_{k=1}^{\tilde{k}} \mathscr{C}^{\{k\}}=\sum_{k=1}^{\tilde{k}}\left(\sum_{i=1}^{n} n n z_{i} \cdot \mathscr{L}_{i}^{\{k\}}\right) .
$$

For the full-mantissa Jacobi, we note that the total computational cost in this scenario is equivalent to the number of nonzeros of $A$, multiplied by the number of iterations $\tilde{k}$ and the value 52 (full mantissa length). For the adaptive-mantissa, the computational cost is obtained incrementally, by simply aggregating the cost of the distinct iterations into $\mathscr{C}^{[1, \tilde{k}]}$, while taking into account that the first three iterations are performed in full accuracy (to derive precise values for $c_{i}$ ).

Figure 4 (left) compares the computational costs (in bits) of the full-mantissa Jacobi and the adaptive-mantissa variant for the three previous values of the extension threshold $\delta$. Concretely, for $\delta=$ 0.1 , the cost of the adaptive-mantissa Jacobi is close to that of the full-Jacobi implementation. This is expected from Figure 3, where this threshold quickly extended the mantissa to 52 bits. Compared with the conventional Jacobi iteration, the differences in favor of 


$$
\delta=0.1
$$
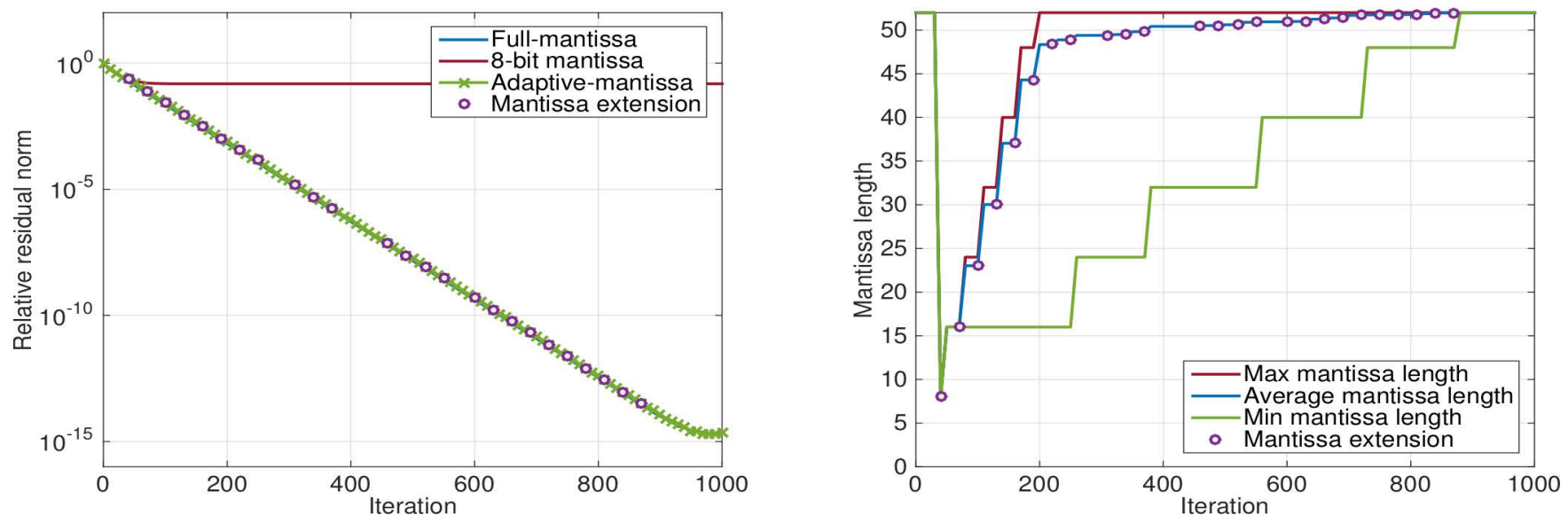

$\delta=0.5$
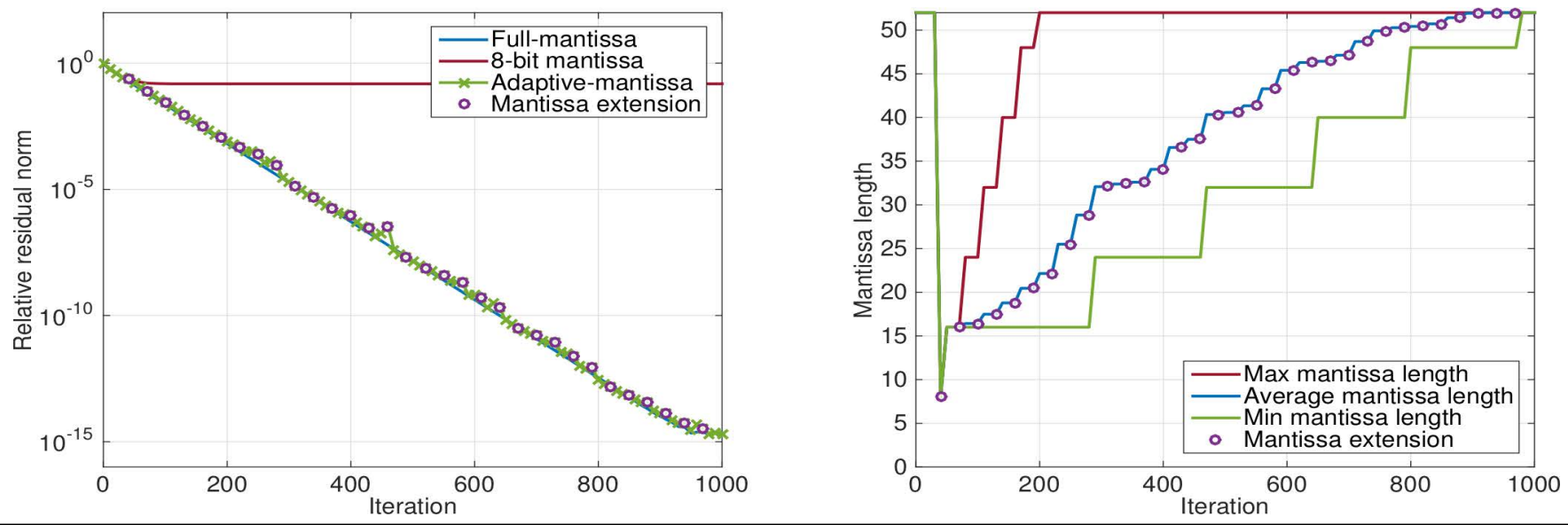

$\delta=0.9$
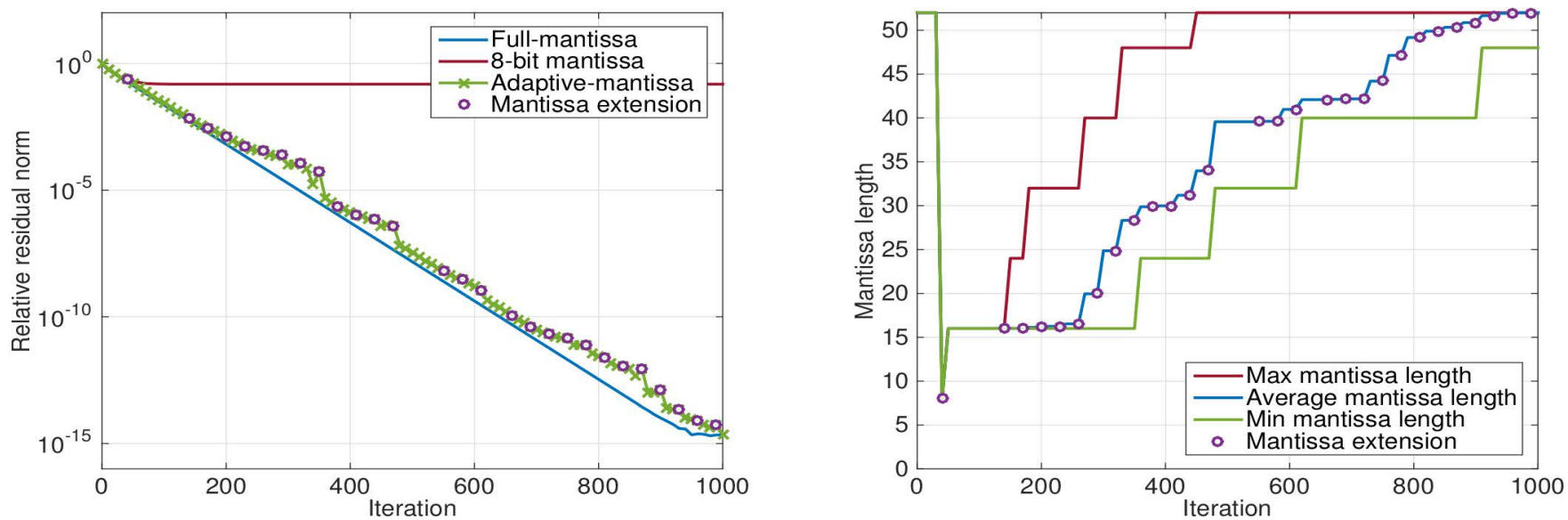

Figure 3: Convergence (left) and behavior of the mantissa length (right) for different values of the threshold $\delta$. The test case is a 27-pt stencil discretization of the 3D Laplace problem using a $16 \times 16 \times 16$ grid. For the adaptive-mantissa Jacobi, $\phi=10$ and $\gamma=8$.

the adaptive-mantissa version grow with the residual threshold (i.e., with the number of iterations). The right-hand side of Figure 4 exposes the computational savings that can be attained with the adaptive-mantissa Jacobi with respect to the conventional Jacobi iteration as a ratio. For this purpose, we now use a fine-grain range of values for $\delta \in(0,1)$, and consider distinct points of the iteration execution, which is stopped when $\mathscr{R}\left(x^{\{k\}}\right)<\tau$ for three different values of the convergence threshold $\tau$. For the selected $\phi=10$ and 

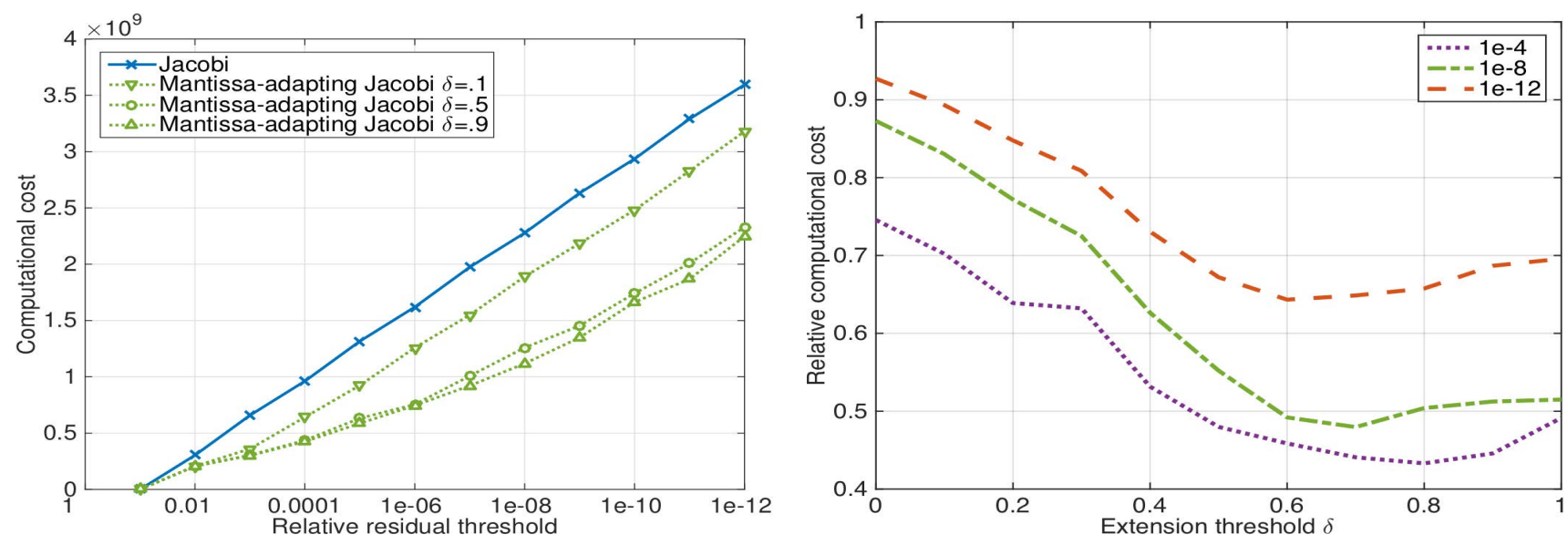

Figure 4: Computational cost of the adaptive-mantissa Jacobi for different values of the threshold $\delta$ (left) and the relative values with respect to the full-mantissa Jacobi for different values of the threshold $\delta$ and three values of the convergence threshold $\tau=10^{-4}, 10^{-8}$, and $10^{-12}$ (right). The test case is a 27 -pt stencil discretization of the $3 \mathrm{D}$ Laplace problem using the $16 \times 16 \times 16$ discretization. For the adaptive-mantissa Jacobi, $\phi=10$ and $\gamma=8$.
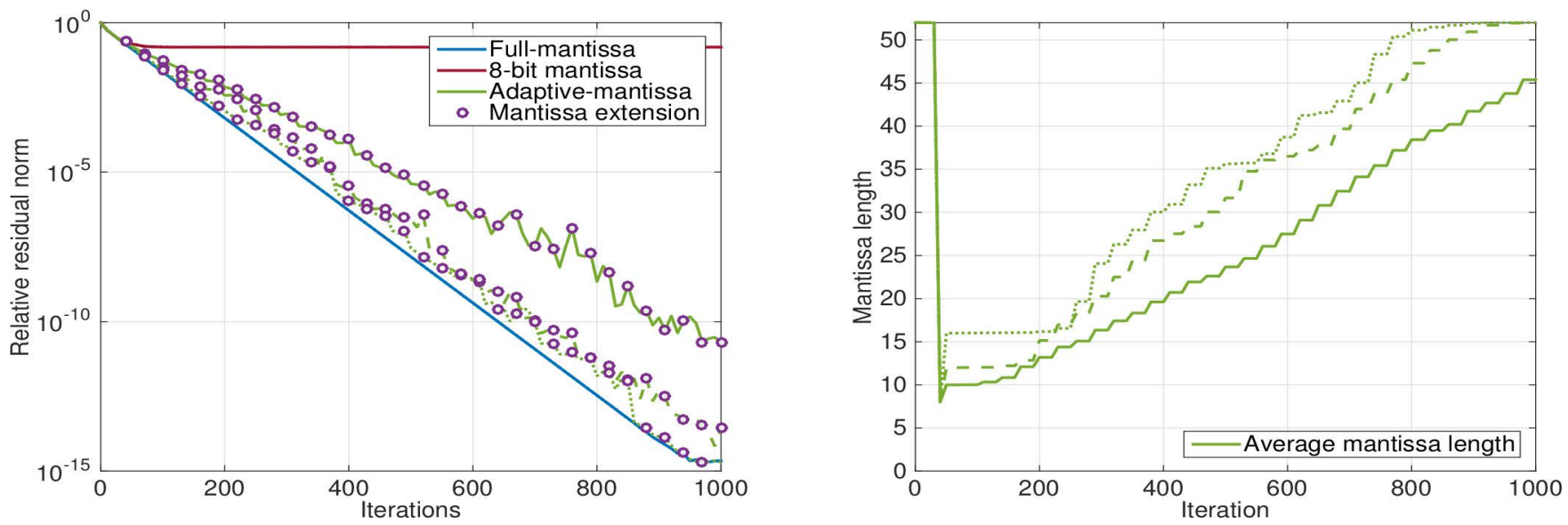

Figure 5: Convergence (left) and behavior of the mantissa length (right) for different values of the bit extension gradient with solid, dashed and dotted lines corresponding to $\gamma=2,4$, and 8, respectively. The test case is a 27-pt stencil discretization of the 3D Laplace problem using a $16 \times 16 \times 16$ grid. For the adaptive-mantissa Jacobi, $\phi=10$ and $\delta=0.8$.

$\gamma=8$, the results in this plot point in the direction of selecting a value for $\delta$ around $0.7-0.8$, which renders computational savings of up to $32 \%$ for $\tau=10^{-12}$ and slightly above $45 \%$ for $\tau=10^{-4}$ and $10^{-8}$.

\subsection{Selecting the bit extension gradient $\gamma$}

Figure 5 reports the effect of the number of bits $\gamma$ that are added to the mantissa (gradient), when the extension condition is violated, for the adaptive-mantissa Jacobi iteration applied to the 3D Laplace problem. Here we fix $\delta=0.8$, which was judged to be a reasonable value in our previous experiment. The results for $\gamma=4$ and 8 show that the convergence rate for the adaptive version is only slightly worse than that of the full-Jacobi iteration, while the use of $\gamma=2$, in combination with $\phi=10$, yields an increase in the length of the mantissa that cannot keep up with the convergence rate of the iteration.

For a fixed checking frequency $\phi$, the threshold $\delta$ for the extension condition, and the extension gradient $\gamma$, may affect each other resulting in a variety of computational costs/savings. Figure 6 con- firms that, for this particular problem, the choices $\delta=0.8$ and $\gamma=8$ are among the best options from the perspective of this metric.

\subsection{Approximate triangular preconditioners}

Jacobi-type iterations have been revisited recently as approximate sparse triangular solvers for incomplete factorization preconditioners such as ILU(0) on architectures providing ample hardware parallelism [10,2]. The traditional exact forward/backward substitution involving the triangular factors are difficult to parallelize, and replacing them with a few inexpensive relaxation steps can result in a significant acceleration of the solver $[2,9]$. However, the scenario for the application of the Jacobi iteration as a solver for these triangular systems differs from those that we considered earlier. In particular, as the incomplete factorizations, in general, only provide a rough approximation, the iteration process is not completed to convergence, but typically stopped after a few steps [10]. Also, the properties of the triangular factors typically result in very fast convergence for a Jacobi-type of solver [2]. This makes the problem of approximate sparse triangular solves inherently unattractive for the 

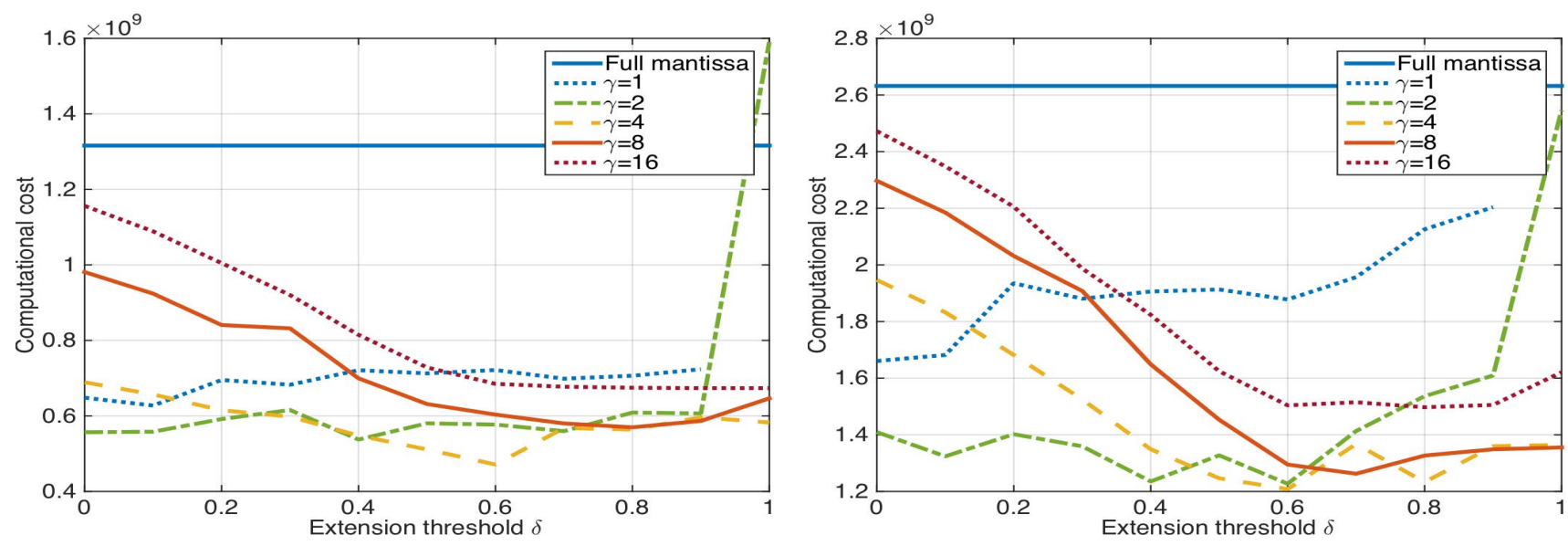

Figure 6: Computational cost of the adaptive-mantissa Jacobi for different values of the threshold $\delta$ and the bit extension gradient $\gamma$, when the iteration is stopped with a convergence criterion $\tau=10^{-4}$ (left) and $\tau=10^{-8}$ (right), respectively. The test case is a 27-pt stencil discretization of the 3D Laplace problem using a $16 \times 16 \times 16$ grid. For the adaptive-mantissa Jacobi, $\phi=10$.

adaptive-mantissa Jacobi. Nonetheless, we target these problems to show that the adaptive-mantissa Jacobi can also be adapted to these requirements, and provide relevant computational savings in a not-so-favorable scenario.

For our practical evaluation, we choose the same benchmarks leveraged in [2]. The systems derive from incomplete factors in an ILU(0) factorization for matrices taken from the University of Florida Matrix Collection (UFMC [11]). Table 1 lists some key characteristics. For each case, we optimized the configuration parameters $\phi, \gamma$, and $\delta$ such that the improvement over the full-mantissa Jacobi is on average maximized over the relative residual thresholds $\tau=10^{-2}, 10^{-4}, \ldots, 10^{-10}$. Note that this is not equivalent to optimizing for each threshold as, due to the nonlinear relation between cost and relative residual threshold (indicated in the left of Figure 4), optimizing for a specific threshold may result in higher benefits.

Table 2 reports the optimal configurations along with the iteration counts and the ratio between the computational cost of the adaptive mantissa and the full-mantissa Jacobi. As expected, using truncated precision (adaptive-mantissa Jacobi) often results in some convergence delay. For those systems exhibiting fast convergence, e.g., the DC problem, this delay is larger than the benefits obtained from the use of a lower precision format. For all other problems, except one case, the adaptive-mantissa strategy yields a variety of gains. Interestingly, the upper triangular factors typically allow for larger savings. A possible explanation is that the diagonal entries of these factors are large compared with the other entries in the respective row. If the diagonal component is large, the demand for higher precision very early results in the stagnation of this component. Otherwise, the information gets blurred by the other components, and the data first propagates through the system before the extension test detects the need for a mantissa extension. For triangular systems, this propagation can be especially slow. Overall, we notice that, despite the unpleasant scenario illustrated by these benchmarks, we were also able to reduce the overall computational cost for sparse triangular solves. However, we recognize that extracting these benefits required an optimization step to adapt the configuration parameters $\phi, \gamma$, and $\delta$ to each specific problem.

\section{VECTOR-WISE ADAPTIVE EXTENSION}

\begin{tabular}{|lc||rrr|}
\hline Matrix & Factor & Dimension & Nonzeros & Condition number \\
\hline \multirow{2}{*}{ CHP } & $L$ & 20,082 & 150,616 & $7.90 \mathrm{e}+05$ \\
& $U$ & 20,082 & 150,616 & $1.75 \mathrm{e}+11$ \\
\hline \multirow{2}{*}{ DC } & $L$ & 116,835 & 441,781 & $6.54 \mathrm{e}+10$ \\
& $U$ & 116,835 & 441,450 & $6.50 \mathrm{e}+09$ \\
\hline \multirow{2}{*}{ LAP } & $L$ & 262,144 & $3,560,572$ & $9.23 \mathrm{e}+06$ \\
& $U$ & 262,144 & $3,560,572$ & $3.65 \mathrm{e}+05$ \\
\hline \multirow{2}{*}{ STO } & $L$ & 213,360 & $1,660,005$ & $1.38 \mathrm{e}+07$ \\
& $U$ & 213,360 & $1,575,003$ & $6.08 \mathrm{e}+07$ \\
\multirow{2}{*}{ VEN } & $L$ & 62,424 & 890,108 & $1.85 \mathrm{e}+07$ \\
& $U$ & 62,424 & 890,108 & $2.51 \mathrm{e}+10$ \\
\hline
\end{tabular}

Table 1: Some properties of the sparse triangular factors.

From the viewpoint of reconfigurable hardware, operating each component with a different precision (even from a very reduced discrete collection of these formats) can be quite complex to implement. Nonetheless, we point out that there is still some benefit to be gained by applying a "vector-wise" working precision (instead of a component-wise strategy), where a violation of the extension condition for one of the components triggers an extension for all of them. This results in a cheaper extension test (likely, only a few of the components have to be checked to decide whether an extension is due), and reduces the complexity of the complexity at the (potential) cost of smaller computational savings. Our results in Figure 7 show that the quasi-optimal parameters for the vector-wise strategy are similar to those identified for the component-wise approach. For this particular problem, and $\phi=10$, we observe that the difference in savings between the component-wise and vector-wise approaches is small, and that the practical values $\delta=0.8$ and $\gamma=8$ are still quite optimal, independently of the selected convergence criterion threshold $\tau$.

\section{CONCLUDING REMARKS}

We have exploited the component-wise contraction property of the Jacobi iteration for sparse linear systems to introduce a formulation of this method that dynamically and individually adapts the mantissa length to save resources, therefore, theoretically saving energy during computation. Concretely, a periodic and cheap extension test determines which components have to be extended by examining the deviations between the expected (theoretical) convergence rate and the observed (experimental) pace, while accommodating small differences due to rounding errors and avoiding 


\begin{tabular}{|c|c|c|c|c|c|c|c|c|c|c|c|c|c|c|}
\hline & \multicolumn{3}{|c|}{$\begin{array}{c}\text { Optimal } \\
\text { configuration }\end{array}$} & \multicolumn{5}{|c|}{$\begin{array}{c}\text { Number of iterations } \\
\text { Adaptive-mantissa Jacobi/full-mantissa Jacobi }\end{array}$} & \multicolumn{5}{|c|}{$\begin{array}{c}\text { Computational cost of adaptive-mantissa } \\
\text { w.r.t. full-mantissa Jacobi }\end{array}$} \\
\hline & & $\phi$ & $\gamma$ & $\delta$ & $10^{-2}$ & $10^{-4}$ & $10^{-6}$ & $10^{-8}$ & $10^{-10}$ & $10^{-2}$ & $10^{-4}$ & $10^{-6}$ & $10^{-8}$ & $10^{-10}$ \\
\hline \multirow{2}{*}{ CHP } & $\bar{L}$ & 7 & 16 & 0.5 & $13 / 11$ & $23 / 20$ & $31 / 29$ & $40 / 38$ & $48 / 46$ & 0.69 & 0.82 & 0.82 & 0.85 & 0.88 \\
\hline & $U$ & 1 & 16 & 0.4 & $12 / 11$ & $22 / 20$ & $29 / 28$ & $37 / 37$ & $45 / 46$ & 0.60 & 0.76 & 0.79 & 0.81 & 0.82 \\
\hline \multirow{2}{*}{ DC } & $L$ & 1 & 32 & 0.8 & $12 / 8$ & $13 / 10$ & $15 / 11$ & $17 / 13$ & $19 / 13$ & 1.31 & 1.13 & 1.21 & 1.17 & 1.34 \\
\hline & $U$ & 1 & 32 & 0.8 & $14 / 8$ & $15 / 10$ & $16 / 11$ & $18 / 12$ & $19 / 13$ & 1.59 & 1.35 & 1.31 & 1.37 & 1.34 \\
\hline \multirow{2}{*}{ LAP } & $L$ & 1 & 32 & 0.5 & $11 / 11$ & $21 / 20$ & $30 / 29$ & $39 / 38$ & $48 / 48$ & 0.59 & 0.74 & 0.77 & 0.79 & 0.79 \\
\hline & $U$ & 1 & 8 & 0.3 & $11 / 11$ & $20 / 20$ & $28 / 29$ & $37 / 38$ & $47 / 48$ & 0.43 & 0.40 & 0.43 & 0.51 & 0.59 \\
\hline \multirow{2}{*}{ STO } & $L$ & 1 & 16 & 0.7 & $10 / 9$ & $21 / 20$ & $31 / 28$ & $38 / 34$ & $46 / 44$ & 0.74 & 0.87 & 0.98 & 1.01 & 0.96 \\
\hline & $U$ & 1 & 8 & 0.1 & $8 / 9$ & $20 / 20$ & $28 / 28$ & $35 / 36$ & $45 / 45$ & 0.51 & 0.65 & 0.75 & 0.78 & 0.85 \\
\hline \multirow[b]{2}{*}{ VEN } & $L$ & 1 & 16 & 0.9 & $25 / 24$ & $43 / 42$ & $60 / 59$ & $74 / 73$ & $86 / 85$ & 0.82 & 0.89 & 0.92 & 0.94 & 0.95 \\
\hline & $U$ & 1 & 8 & 0.6 & $17 / 17$ & $36 / 35$ & $53 / 52$ & $68 / 68$ & $82 / 82$ & 0.54 & 0.77 & 0.85 & 0.87 & 0.89 \\
\hline
\end{tabular}

Table 2: Comparison of convergence delay and computational cost reduction for the configuration of the adaptive-mantissa Jacobi optimized over the considered relative residual thresholds $\tau$.
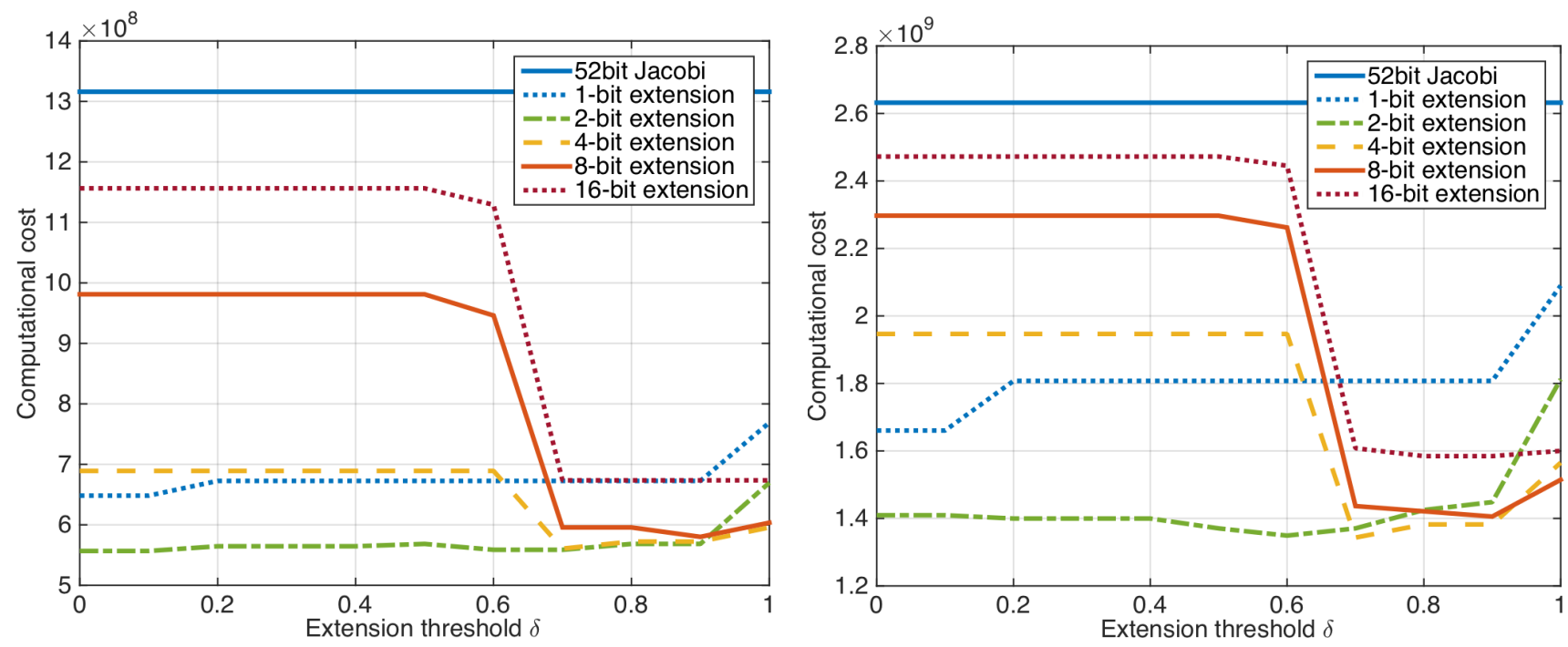

Figure 7: Computational cost of the adaptive-mantissa Jacobi using a "vector-wise" precision extension for different values of the threshold $\delta$ and the bit extension gradient $\gamma$, when the iteration is stopped with a convergence criterion $\tau=10^{-4}$ (left) and $\tau=10^{-8}$ (right), respectively. The test case is 27 -pt stencil discretization of the 3D Laplace problem using a $16 \times 16 \times 16$ grid. For the adaptive-mantissa Jacobi, $\phi=10$.

stagnation. The parameters of the approach allow us to tune the periodicity, extension threshold, and bit extension gradient for each problem case.

The experimental results with a standard 3D Laplace benchmark, and a collection of sparse triangular linear systems appearing in the iterative application of ILU preconditioners, reveal that the potential savings for the component-wise adaptive-Jacobi iteration can reach up to $60 \%$. Theoretically, a reduction of $50 \%$ could also be achievable by monitoring the residual over the iteration process, but this requires expensive residual computations with a computational cost similar to the iteration.

In practice, we envision that this approach can be applied to accelerate the computations and/or reduce the energy cost. For this purpose, we propose a realization using reconfigurable hardware for a vector-wise adaptive-Jacobi variant of the idea which simultaneously adapts the mantissa length for all components and leverages a small collection of discrete mantissa lengths (e.g., 8, 16, $24, \ldots, 48,52$; or $16,32,48$ and 52 bits).

\section{Acknowledgments}

This work was partly funded by the U.S. Department of Energy Office of Science, Office of Advanced Scientific Computing Research, Applied Mathematics program under Award Number DE-
SC-0010042, and the Russian Scientific Foundation, Agreement N14-11-00190. E. S. Quintana-Ortí was supported by projects TIN201123283 and TIN2014-53495-R of the Spanish Ministerio de Economía y Competitividad.

\section{REFERENCES}

[1] The top500 list, 2015. http://www.top500.org. Last access: July 2015.

[2] H. Anzt, E. Chow, and J. Dongarra. Iterative sparse triangular solves for preconditioning. In Euro-Par 2015: Parallel Processing, volume 9233 of Lecture Notes in Computer Science, pages 650-661. 2015.

[3] H. Anzt, J. Dongarra, and E. S. Quintana-Ortí. Tuning stationary iterative solvers for fault resilience. In Workshop on Latest Advances in Scalable Algorithms for Large-Scale Systems, ScalA, 2015. DOI: 10.1145/2832080.2832081.

[4] H. Anzt and E. S. Quintana-Ortí. Improving the energy efficiency of sparse linear system solvers on multicore and manycore systems. Philosophical Trans. Royal Society of London A: Mathematical, Physical and Engineering Sciences, 372(2018), 2014.

[5] H. Anzt, S. Tomov, J. Dongarra, and V. Heuveline. A block-asynchronous relaxation method for graphics 
processing units. Journal of Parallel and Distributed Computing, 73(12):1613-1626, 2013.

[6] S. Barrachina, M. Castillo, F. D. Igual, R. Mayo, E. S. Quintana-Ortí, and G. Quintana-Ortí. Exploiting the capabilities of modern GPUs for dense matrix computations. Concurrency and Computation: Practice and Experience, 21(18):2457-2477, 2009.

[7] A. Buttari, J. Dongarra, J. Langou, J. Langou, P. Luszczek, and J. Kurzak. Mixed precision iterative refinement techniques for the solution of dense linear systems. Int. J. High Performance Computing Applications, 21(4):457-466, 2007.

[8] Z. Chen. Online-ABFT: An online algorithm based fault tolerance scheme for soft error detection in iterative methods. In Proc. 18th ACM SIGPLAN Symp. Principles and Practice of Parallel Programming, PPoPP'13, pages 167-176, 2013.

[9] E. Chow, H. Anzt, and J. Dongarra. Asynchronous iterative algorithm for computing incomplete factorizations on GPUs. In Lecture Notes in Computer Science, volume 9137, pages 1-16, 2015.

[10] E. Chow and A. Patel. Fine-grained parallel incomplete LU factorization. SIAM Journal on Scientific Computing, 37(2):C169-C193, 2015.

[11] T. A. Davis. The University of Florida Sparse Matrix Collection. NA DIGEST, 92, 1994.

[12] R. Dennard, F. Gaensslen, V. Rideout, E. Bassous, and A. LeBlanc. Design of ion-implanted MOSFET's with very small physical dimensions. IEEE J. Solid-State Circuits, 9(5):256-268, 1974.

[13] J. Elliott, M. Hoemmen, and F. Mueller. Evaluating the impact of SDC on the GMRES iterative solver. In Proc. 2014 IEEE 28th Int. Parallel and Distributed Processing Symp., IPDPS'14, pages 1193-1202, 2014.

[14] H. Esmaeilzadeh, E. Blem, R. St. Amant, K. Sankaralingam, and D. Burger. Dark silicon and the end of multicore scaling. In Proc. 38th Annual Int. Symp. Computer architecture, ISCA'11, pages 365-376, 2011.

[15] P. Gschwandtner, C. Chalios, D. Nikolopoulos, H. Vandierendonck, and T. Fahringer. On the potential of significance-driven execution for energy-aware HPC. Computer Science-Research and Development, 30:197-206, 2015.

[16] N. Hardavellas, M. Ferdman, B. Falsafi, and A. Ailamaki. Toward Dark Silicon in servers. Micro, IEEE, 31(4):6-15, 2011.

[17] N. Higham. Accuracy and Stability of Numerical Algorithms.
Philadelphia, PA, 1996.

[18] M. Hoemmen and M. A. Heroux. Fault-tolerant iterative methods via selective reliability. In Proc. 2011 Int. Conf. for High Performance Computing, Networking, Storage and Analysis (SC), 2011.

[19] W. Kahan. Lecture notes on the status of IEEE standard 754 for binary floating-point arithmetic, 1997. http://www.eecs. berkeley.edu/ wkahan/ieee754status/IEEE754.PDF.

[20] P. Kogge et al. ExaScale computing study: Technology challenges in achieving ExaScale systems, 2008. http://users. ece.gatech.edu/ mrichard/ExascaleComputingStudyReports/ exascale_final_report_100208.pdf.

[21] J. F. Lavignon et al. ETP4HPC strategic research agenda achieving HPC leadership in Europe, 2013. http://www.etp4hpc.eu/.

[22] S. Liu, K. Pattabiraman, T. Moscibroda, and B. G. Zorn. Flikker: Saving DRAM refresh-power through critical data partitioning. In Proc. Sixteenth International Conference on Architectural Support for Programming Languages and Operating Systems, ASPLOS XVI, pages 213-224, 2011.

[23] G. Moore. Cramming more components onto integrated circuits. Electronics, 38(8):114-117, 1965.

[24] A. Rahimi, A. Marongiu, R. Gupta, and L. Benini. A variability-aware OpenMP environment for efficient execution of accuracy-configurable computation on shared-FPU processor clusters. In Hardware/Software Codesign and System Synthesis (CODES+ISSS), 2013 International Conference on, pages 1-10, 2013.

[25] Y. Saad. Iterative Methods for Sparse Linear Systems. SIAM, 2003.

[26] H. F.-W. Sadrozinski and J. Wu. Applications of Field-Programmable Gate Arrays in Scientific Research. CRC Press, 2010.

[27] A. Sampson, W. Dietl, E. Fortuna, D. Gnanapragasam, L. Ceze, and D. Grossman. EnerJ: Approximate data types for safe and general low-power computation. In Proc. 32Nd ACM SIGPLAN Conference on Programming Language Design and Implementation, PLDI'11, pages 164-174, 2011.

[28] P. Sao and R. Vuduc. Self-stabilizing iterative solvers. In Workshop Latest Advances in Scalable Algorithms for Large-Scale Systems, pages 4:1-4:8, 2013.

[29] Semiconductor Industries Association. International Technology Roadmap for Semiconductors, 2013.

http://www.itrs.net/. Last access: July 2015. 